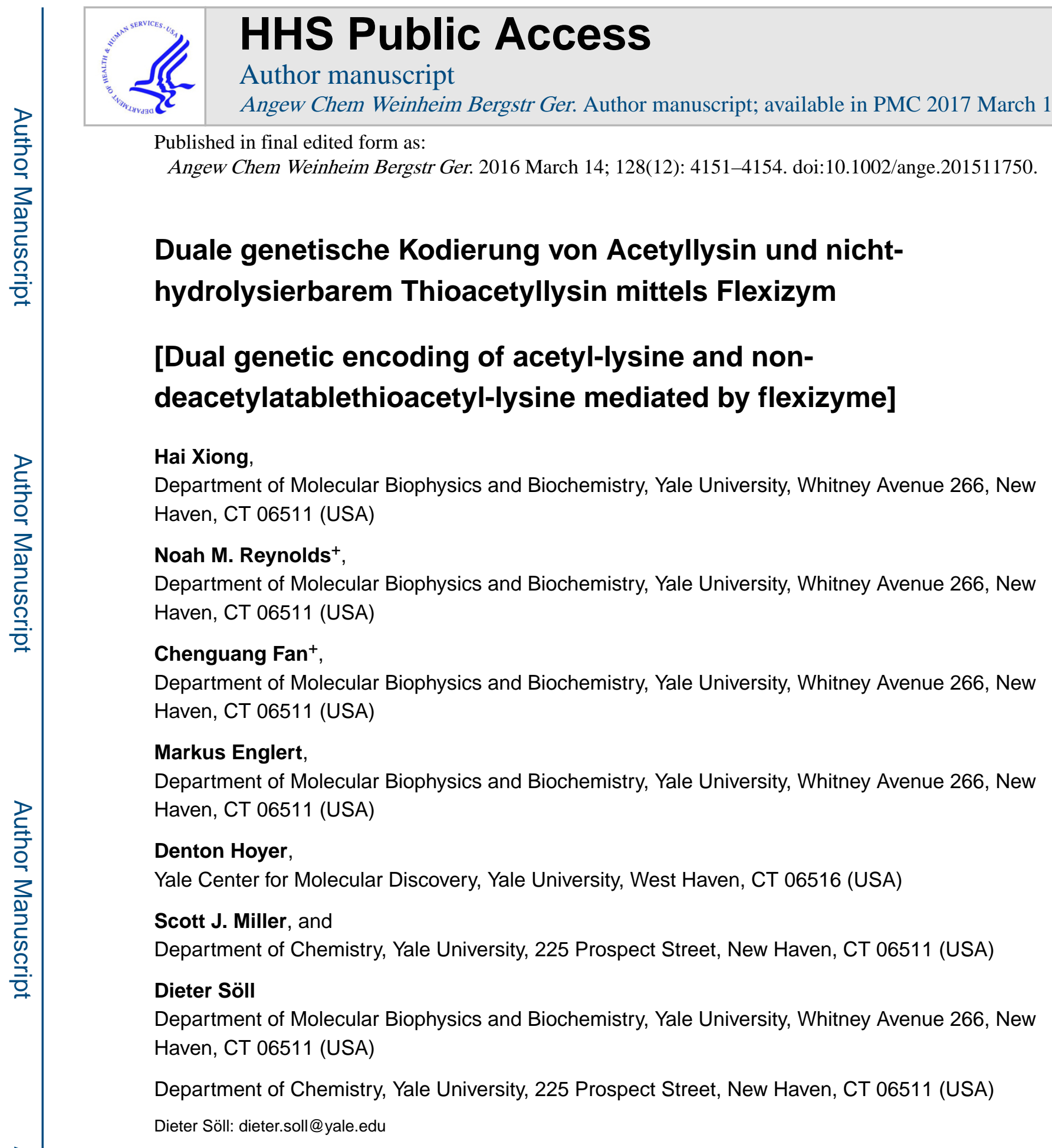

Abstract

Die Acetylierung von Lysin-Bausteinen ist eine wichtige posttranslationale Proteinmodifikation. Die Lysin-Acetylierung in Histonproteinen und deren Interaktion mit anderen posttranslationalen Modifikationen in Histon- und nicht-Histon-Proteinen ist essentiell bei der DNA-Replikation, DNA-Reparatur und Transkriptionsregulation. Wir verwendeten die Flexizym-Technologie, um die Aminos uren Acetyllysin (AcK) und nicht-hydrolysierbares Thioacetyllysin (Thio-AcK) in

\footnotetext{
Correspondence to: Dieter Söll, dieter. soll@yale. edu.

${ }^{+}$Diese Autoren haben zu gleichen Teilen zu der Arbeit beigetragen.
} 
vitro in intakte Proteine einzubauen. ThioAcK und AcK wurden in humanes Histon H3 positionsspezifisch in-korporiert. Dies erfolgte entweder individuell oder paarweise bei verschiedenen Lysin-Positionen innerhalb des humanen Histon H3. Die Thioacetylgruppe im Histon H3 konnte nicht durch die Histon-Deacetylase Sirtuin (Typ 1) abgespalten werden, wie durch diese Studie gezeigt wurde. Diese Methode des AcK- und ThioAcK-Einbaus stellt ein wichtiges Werkzeug für die Untersuchung der Protein-Acetylierung sowie deren Rolle in der Interaktion mit weiteren posttranlationalen Modifizierungen dar.

\section{Präzise Schnitte}

Die Flexizym-Technik wurde verwendet, um Acetyllysin und nicht-hydrolysierbares Thioacetyllysin entweder einzeln oder paarweise positionsspezifisch in humanes Histon H3 einzubauen (siehe Schema). Es wurde gefunden, dass die Thioacetylgruppe des modifizierten Histon H3 nicht durch die Histon-Deacetylase Sirtuin abgespalten wird.

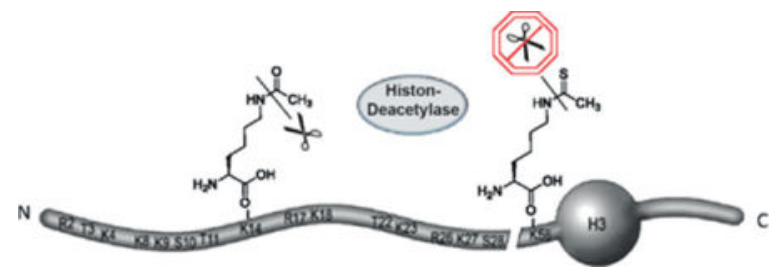

Posttranslationale Modifikationen (PTMs) der aminoterminalen Enden von Histonen dienen als epigenetische Indikatoren für die Chromatin-Struktur, die mit der Genregulation, der DNA-Reparatur und der transkriptionellen Stilllegung der Genexpression assoziiert ist. ${ }^{[1]}$ Die Acetylierung von Lysin-Bausteinen innerhalb von Histonen korreliert mit den dynamischen Übergängen der Chromatin-Struktur und -Funktion, der transkriptionellen Aktivierung der Genexpression sowie der DNA-Replikation. ${ }^{[1 \mathrm{a}, \mathrm{c}]}$ Die Interaktion von LysinAcetylierung innerhalb von Histonen und anderen PTMs ist von essentieller Bedeutung für die vererbbaren epigenetischen Programme, die über die Chromatin-Struktur gestaltet und gesteuert werden. ${ }^{[1 f, 2]}$ Die Funktion und Regulation des Histon-Acetylierungsmusters wird durch die kumulative Wirkung von Histon-Acetyltransferasen (HATs) und HistonDeacetylasen (HDACs) bewirkt; HATs und HDACs modifizieren mehrere Lysin-Bausteine, zudem werden etliche Lysin-Positionen von mehreren Enzymen umgesetzt. ${ }^{[3]}$ Studien der PTMs mit analogen nicht-hydrolysierbaren Bausteinen stellen ein neuartiges Werkzeug für die Analyse von Enzymen dar, die diese Modifikationen in vivo modulieren. Der $\mathrm{N}^{\varepsilon}$ Thioacetyllysin-Baustein ist ein Derivat von Acetyllysin, das durch eine einzige atomare Substitution erhalten wird, und besitzt die ähnlichsten chemischen Eigenschaften unter den Acetyllysin-Analoga. ${ }^{[4]}$ Bisher wurde ThioAcK in kurze Peptide mittels Festphasenpeptidsynthese eingebaut, deren Sequenz von den p53- und a-Tubulin-Proteinen abgeleitet wurden. Solche Peptide stellten einerseits effektive Inhibitoren des menschlichen Sirtuin-Proteins dar und wurden aufgrund der nicht-hydrolysierbaren Eigenschaft des ThioAcK-Bausteins auch als multifunktionale Sonden eingesetzt. ${ }^{[4,5]}$ Jedoch wurde ThioAcK noch nicht in intakte Proteine eingebaut, sodass die Verwendung von ThioAcK bei Histonmodifizierungen bisher eingeschränkt war. 
Der Einbau von nicht-kanonischen Aminosäuren (nkAS) in Peptide und Proteine wurde mittels verschiedener Methoden erreicht, darunter Halbsynthesen von Proteine, die mit chemischer Proteinligation kombiniert wurden, sowie die Erweiterung des genetischen Codes mit orthogonalen Aminoacyl-tRNA-Synthase-Paaren [(asRS).tRNA] ${ }^{[6]}$ Die gleichzeitige Suppression der UAG- und UGA-Codons wurde mittels zweier Paare, nämlich Pyrrolysyl-tRNA-Synthase-tRNA ${ }^{\mathrm{Pyl}}$ und $M$. jannaschii Tyrosyl-tRNA-Synthase.tRNA ${ }^{\mathrm{Tyr}}$, ermöglicht. Zudem wurde auch die Suppression von Quadruplett-Codons verwendet, um verschiedene nkAS in ein Protein einzubauen. ${ }^{[7]}$

Die Acylierung von tRNAs mit nkAS kann zum einen mittels orthogonaler asRS.tRNAPaare oder durch die chemische Acylierung eines Dinukleotids mit anschließender Ligation an eine verkürzte tRNA erfolgen; jedoch erfordern beide Methoden mehrstufige Experimente, die mit erheblichen Arbeits- und Materialkosten verbunden sind. ${ }^{[8]}$ Eine allgemeine und attraktive Methode zur Bildung von Aminoacyl-tRNAs wurde von Suga und Mitarbeitern entwickelt. ${ }^{[9]}$ Eine katalytisch aktive RNA (das Flexizym) erkennt und belädt die tRNA mit einer Aminosäure (AS), die zuvor aktiviert wurde. Aromatische AS können in Form des Cyanmethylesters als Substrat dienen. Eine weitere Verbesserung der ASAktivierung führte zu der Dinitro-Flexizym-Technologie (dFx), der dazugehörige 3,5Dinitrobenzylester aktiviert jetzt die AS unabhängig von der AS-Seitenkette. ${ }^{[9 a]}$

Die Flexizym-Technologie wurde bereits zur Synthese von Peptiden verwendet, die vom aminoterminalen Ende des Histon $\mathrm{H} 3$ abgeleitet wurden und zum Einbau von $N$-Methyllysin oder Acetyllysin führten. Über die Verwendung der Flexizym-Technologie für die Expression von intakten Histon-Proteinen wurde jedoch noch nicht berichtet. ${ }^{[9 b]}$

In dieser Studie wurden intakte Histon-H3-Proteine unter Verwendung von dFx exprimiert, die zum Einbau von AcK und nicht-deacetylierbarem ThioAcK führten. Hierfür wurden die 3,5-Dinitrobenzylester von $\mathrm{N}^{\varepsilon}$-Acetyllysin (AcK-DBE, 3), $\mathrm{N}^{\varepsilon}$-Thioacetyllysin (ThioAcKDBE, 4) und $\mathrm{N}^{\varepsilon}$-Selenoacetyllysin (SeAcK-DBE, 5) verwendet (Abbildung 1). SeAcK wurde synthetisiert, um die Effektivität und den allgemeinen Nutzen unserer Methode zu testen. AcK, ThioAcK und SeAcK unterscheiden sich in der Anwesenheit von Sauerstoff, Schwefel und Selen in der Carbonyl-Seitengruppe. Die neuen chemischen Komponenten ThioAcK-DBE und SeAcK-DBE wurden synthetisiert und mittels ${ }^{1} \mathrm{H}-\mathrm{NMR}-{ }^{-}{ }^{13} \mathrm{C}-\mathrm{NMR}$ Spektroskopie sowie HPLC-Massenspektrometrie (MS) charakterisiert (Hintergrundinformationen (SI)). Val-DBE (1), Tyr-DBE (2) und AcK-DBE (3), die mit leichten Veränderungen nach Literaturvorgaben synthetisiert wurden, ${ }^{[9 \mathrm{a}]}$ dienten als Kontrolle für tRNA-Acylierung durch dFx.

Die Flexiyzme und die davon abgeleiteten Derivate erkennen das 3'-Ende der tRNA, das RCCA-3' aufweisen muss (R steht für G oder A an der Position 73). Daher wurde bei der tRNA ${ }^{\text {Sep }}$ das $3^{\prime}$-Ende durch die Mutation U73A zu UCCA-3' verändert (U73A-tRNA ${ }^{\text {Sep, }}$ Abbildung S1 in den SI). Wir testeten die Aminoacylierungseffizienz von tRNA ${ }^{\text {Sep }}$ oder U73A-tRNA ${ }^{\text {Sep }}$ mit den Komponenten 1 und 2 mittels des dFx-Systems; alle Kombinationen zeigten eine Beladungseffizienz im Bereich von $36-45 \%$ auf (Abbildung S2). Bisherige Studien der Flexizym-Technologie wurden mit tRNA ${ }^{\mathrm{Asn}}$, tRNA ${ }^{\text {fMetE }}$ und Mj-tRNA ${ }^{\mathrm{Tyr}}$ CUA 
durchgeführt. Daher wurden diese tRNAs als Kontrollen für die Beladung mit unseren synthetisierten Aminosäurebenzylestern verwendet (Abbildung S2).

Innerhalb der U73A-tRNA ${ }^{\text {Sep }}$ wurde die Anticodonsequenz verändert, sodass diese UAA, UGA sowie Quadruplett-Codons lesen können. Alle Kombinationen der tRNA ${ }^{\text {Sep-Mutanten }}$ und der aktivierten Aminosäuren resultierten unter Verwendung von $\mathrm{dFx}$ in signifikanten Aminoacylierungsraten, die sich im Bereich von 26-38 \% befinden (Abbildung S2). Das Invitro-Translationssystem PUREexpress (NEB) wurde in Abwesenheit von Releasefaktor 1 (PURE $\triangle R F 1$ ) für die Suppresion von UAG eingesetzt. Hierfür wurde U73A-tRNA ${ }^{\text {Sep }}$ CUA mittels dFx beladen und für den Einbau von AcK, ThioAcK und SeAcK getestet. Als Reporter diente das Protein "superfolder-GFP", dessen kodierende mRNA ein UAG-Codon an der Position 151 aufweist (sfGFP-Y151TAG). Die Effizienz der UAG-Suppression wurde über die Fluoreszenzintensität im Vergleich zum Wildtyp-sfGFP ermittelt und beläuft sich auf $15 \%$ für den Einbau von Val, $32 \%$ für Tyr, $41 \%$ für AcK, $37 \%$ für ThioAcK und $26 \%$ für SeAcK (Abbildungen S3-S5 und Tabellen S1-S2).

Der Einbau von AcK, ThioAcK und SeAcK in humanes Histon H3 erfolgte an den Positionen K9 und K56. Die Proteinexpression fand im PURE-ARF1-System mittels UAGSuppression und U73A-tRNA ${ }^{\mathrm{Sep}}{ }_{\mathrm{CUA}}$ statt, die mittels $\mathrm{dFx}$ aminoacyliert wurde. Für humanes Histon $\mathrm{H} 3$ sind Antikörper erhältlich, die positionsspezifisch die Acetylierung von K9 oder K56 erkennen; sie wiesen eine starke immunologische Kreuzreaktion auf, wenn ThioAcK dort eingebaut wurde (Abbildung 2A). Ein weiteres Experiment zeigte auf, dass diese positionsspezifischen AcK-Antikörper eine ähnliche Affinität zu AcK und ThioAcK aufweisen (Abbildung S14). Der Einbau von AcK und ThioAcK in humanes Histon H3 an Position K56 wurde mittels Tandem-Massenspektrometrie (MS/MS) bestätigt (Abbildungen 2B und C). Jedoch war eine Detektion der AcK- und ThioAcK-Modifizierung an der Position K9 von humanem Histon H3 mittels MS/MS nicht möglich. Die Proteinsequenz von Histon $\mathrm{H} 3$ enthält viele Lysinreste nahe $\mathrm{K}$, sodass aus der tryptischen Hydrolyse ein kurzes und polares Peptid mit K9 entsteht. Derartige Peptide sind massenspektrometrisch sehr schwer zu detektieren. ${ }^{[6 b]}$ Nach dem Einbau von SeAcK in Histon H3 war diese Modifizierung nicht durch MS/MS-Analyse nachweisbar. Anstelle von SeAcK wurde AcK nachgewiesen, sodass von einer chemischen Modifikation bei der Probenaufarbeitung ausgegangen wird. ThioAcK wurde über die UAG- und UGA-Codons sehr effizient eingebaut; jedoch war die Inkorporation von ThioAcK über UAA bei K56 nicht erfolgreich (Abbildung 2A). Im Allgemeinen wurde in dieser Studie herausgefunden, dass der Einbau von ThioAcK mittels UAA vom mRNA-Kontext abhängt und insgesamt schwach verläuft.

Wie in der Literatur beschrieben, wurden bereits vom Protein p53 abgeleitete Peptide mittels Peptidfestphasensynthese unter Einbau von ThioAcK hergestellt. Diese Peptide mit ThioAcK widerstanden der Deacetylierungsreaktion, die normalerweise vom Enzym SIRT1 katalysiert wird. ${ }^{[4,10]}$ Jedoch gibt es kaum Quellen für Deacetylierungsstudien unter Verwendung von intakten Proteinen mit eingebauten nkAS; diese waren bisher aufgrund ihrer aufwendigen Synthese schwer zugänglich. Zwar wurden chemisch modifizierte Peptide als Mimetika von Histon-Proteinen verwendet, diese erlaubten jedoch kaum, die miteinander verschachtelten Interaktionen von verschiedenen PTM-Domänen aufzuzeigen, die bei intakten Histonen vorliegen. ${ }^{[11]}$ In vorliegender Studie wurde der erfolgreiche Einbau von 
AcK und ThioAcK in Histon $\mathrm{H} 3$ aufgezeigt. Wir richteten daher die Aufmerksamkeit auf die Deacetylierungsenzyme (HDACs) und deren Einwirkung auf AcK in intaken Proteinen. Wir entschieden uns für das humane SIRT1-Protein, das als Enzym der Klasse III die NAD ${ }^{+}$abhängige Deacetylierung von AcK-Bausteinen in Proteinen katalysiert. Das intakte Histon H3 mit AcK oder ThioAcK (Position K56) wurde als Substrat für SIRT1 verwendet. Die zeitabhängige Studie bei $37^{\circ} \mathrm{C}$ resultierte im $70 \%$ igen Abbau des AcK nach 90 Minuten, während die ThioAcK-Modifizierung stabil blieb. Dieses Ergebnis belegt die Resistenz der K56ThioAcK-Modifizierung im humanen Histon H3 gegenüber der SIRT1-Deacetylase.

Eine systematische Studie der ThioAcK-Inkorporation in humanes Histon H3 (Positionen K9, K14, K36 und K56) wurde unter Verwendung verschiedener Stop-Codons ausgewertet. Der ThioAcK-Einbau wurde mithilfe eines kontextunspezifischen AcK-Antikörpers und Western Blot analysiert. Die höchste Einbaurate wurde über das UAG-Codon bei K14 und über UGA bei K56 erzielt (Abbildungen 3A, S7 und S8).

Eine weitere Validierung erfolgte durch positionsspezifische Antikörper, die gegen Histon H3 mit der AcK14- oder AcK56-Modifizierung gerichtet sind (Abbildungen 3A und S9). Für eine duale Kodierung von AcK oder ThioAcK in humanes Histon H3 wurden die Positionen K14/K56 und K36/K56 gewählt. Wir konnten aufzeigen, dass die höchste Einbaurate der gleichen nkAS (AcK oder ThioAcK) unter der gegensätzlichen Verwendung von UGA versus UAG erzielt wurde (Abbildungen 3 und S7). Das dual codierte ThioAcKHiston $\mathrm{H} 3$ wurde mittels MS/MS analysiert, was den korrekten Einbau an den Positionen K36/K56 und K14/K56 aufzeigte (Abbildungen S12 und S13, Tabellen S5 und S6).

Nach dem dualen Einbau von entweder AcK oder ThioAcK versuchten wir den gleichzeitigen Einbau eines AcK mit einem ThioAcK in intaktes humanes Histon H3. Da die Einbaurate in Histon H3 über K14 höher als über K36 war (Abbildung S7), wurden beide Positionen mittels UAG und UGA rekodiert (Abbildung 3A). Alle Permutationen wurden getestet: ThioAcK über UGA bei K14 und AcK über UAG bei K56 (K14ThioAcK(UGA)/ $\mathrm{K} 56 \mathrm{AcK}(\mathrm{UAG})$ ), AcK über UGA bei K14 und ThioAcK über UAG bei K56 (K14Ack(UGA)/K56ThioAcK(UAG)), ThioAcK über UAG bei K14 und AcK über UGA bei K56 (K14ThioAcK(UAG)/K56AcK(UGA)), AcK über UAG bei K14 und ThioAcK über UGA bei K56 (K14AcK(UAG)/K56ThioAcK(UGA)). Die Western-Blot-Analyse zeigte eine signifikante Kreuzreaktion bei fast allen Kombinationen (Abbildungen 3A, S8 und S9). Das Histon H3 K14ThioAcK/K56AcK wurde tryptisch hydrolysiert und mittels MS/MS analysiert. Das sekundäre Fragmentierungsmuster zeigt die korrekten Massen für die 14ThioAcK- und 56AcK-Modifizierungen (Abbildung 3B).

In dieser Studie wurde das erste Beispiel für die Synthese von intaktem humanem Histon H3 mit einer Kombination aus ThioAcK- und AcK-Modifizierungen aufgezeigt. Die Verwendung dieser und ähnlicher Histonvarianten sollte zu einem tieferen Verständnis des wichtigen Prozesses der Histon-Acetylierung und -Deacetylierung führen. $\left.{ }^{[4,9 b, 12}\right]$

\section{Supplementary Material}

Refer to Web version on PubMed Central for supplementary material. 


\title{
Acknowledgments
}

\author{
Danksagung
}

Wir danken den Mitarbeitern der Arbeitsgruppe von Prof Söll für die hilfreichen Kommentare. Diese Arbeit wurde durch die National Institutes of Health (GM22854 an D.S.; GM68649 an S.J.M.; AI119813 an C.F.) und die Defense Advanced Research Agency (Vertrag N66001-12-C4211, D.S.) finanziell gefördert.

\section{References}

1. a) Tsubota T, Berndsen CE, Erkmann JA, Smith CL, Yang L, Freitas MA, Denu JM, Kaufman PD. Mol Cell. 2007; 25:703-712. [PubMed: 17320445] b) Chen CC, Carson JJ, Feser J, Tamburini B, Zabaronick S, Linger J, Tyler JK. Cell. 2008; 134:231-243. [PubMed: 18662539] c) Choudhary C, Kumar C, Gnad F, Nielsen ML, Rehman M, Walther TC, Olsen JV, Mann M. Science. 2009; 325:834-840. [PubMed: 19608861] d) Park HS, Hohn MJ, Umehara T, Guo LT, Osborne EM, Benner J, Noren CJ, Rinehart J, Söll D. Science. 2011; 333:1151-1154. [PubMed: 21868676] e) Duan MR, Smerdon MJ. J Biol Chem. 2014; 289:8353-8363. [PubMed: 24515106] f) Yang XJ, Seto E. Mol Cell. 2008; 31:449-461. [PubMed: 18722172]

2. Lee JS, Smith E, Shilatifard A. Cell. 2010; 142:682-685. [PubMed: 20813257]

3. a) Lee KK, Workman JL. Nat Rev Mol Cell Biol. 2007; 8:284-295. [PubMed: 17380162] b) Dhalluin C, Carlson JE, Zeng L, He C, Aggarwal AK, Zhou MM. Nature. 1999; 399:491-496. [PubMed: 10365964]

4. Fatkins DG, Monnot AD, Zheng W. Bioorg Med Chem Lett. 2006; 16:3651-3656. [PubMed: 16697640]

5. a) Mellini P, Kokkola T, Suuronen T, Salo HS, Tolvanen L, Mai A, Lahtela-Kakkonen M, Jarho EM. J Med Chem. 2013; 56:6681-6695. [PubMed: 23927550] b) Kiviranta PH, Suuronen T, Wallen EAA, Leppanen J, Tervonen J, Kyrylenko S, Salminen A, Poso A, Jarho EM. J Med Chem. 2009; 52:2153-2156. [PubMed: 19296597]

6. a) Liu CC, Schultz PG. Annu Rev Biochem. 2010; 79:413-444. [PubMed: 20307192] b) Gattner MJ, Vrabel M, Carell T. Chem Commun. 2013; 49:379-381.c) Zhang Z, Tan M, Xie Z, Dai L, Chen Y, Zhao Y. Nat Chem Biol. 2011; 7:58-63. [PubMed: 21151122] d) Kim CH, Kang M, Kim HJ, Chatterjee A, Schultz PG. Angew Chem Int Ed. 2012; 51:7246-7249.Angew Chem. 2012; 124:7358-7361.e) Wan W, Tharp JM, Liu WR. Biochim Biophys Acta Proteins Proteomics. 2014; 1844:1059-1070.

7. a) Sachdeva A, Wang K, Elliott T, Chin JW. J Am Chem Soc. 2014; 136:7785-7788. [PubMed: 24857040] b) Wang K, Sachdeva A, Cox DJ, Wilf NM, Lang K, Wallace S, Mehl RA, Chin JW. Nat Chem. 2014; 6:393-403. [PubMed: 24755590] c) Wan W, Huang Y, Wang Z, Russell WK, Pai PJ, Russell DH, Liu WR. Angew Chem Int Ed. 2010; 49:3211-3214.Angew Chem. 2010; 122:3279_ 3282.d) Neumann H, Wang K, Davis L, Garcia-Alai M, Chin JW. Nature. 2010; 464:441-444. [PubMed: 20154731]

8. a) Hecht SM, Alford BL, Kuroda Y, Kitano S. J Biol Chem. 1978; 253:4517-4520. [PubMed: 248056] b) Wang L, Brock A, Herberich B, Schultz PG. Science. 2001; 292:498-500. [PubMed: 11313494]

9. a) Murakami H, Ohta A, Ashigai H, Suga H. Nat Methods. 2006; 3:357-359. [PubMed: 16628205] b) Kang TJ, Yuzawa S, Suga H. Chem Biol. 2008; 15:1166-1174. [PubMed: 19022177] c) Goto Y, Katoh T, Suga H. Nat Protoc. 2011; 6:779-790. [PubMed: 21637198] d) Bessho Y, Hodgson DR, Suga H. Nat Biotechnol. 2002; 20:723-728. [PubMed: 12089559] e) Terasaka N, Hayashi G, Katoh T, Suga H. Nat Chem Biol. 2014; 10:555-557. [PubMed: 24907900]

10. a) Hirsch BM, Zheng W. Mol Biosyst. 2011; 7:16-28. [PubMed: 20842312] b) Vaziri H, Dessain SK, Eagon EN, Imai SI, Frye RA, Pandita TK, Guarente L, Weinberg RA. Cell. 2001; 107:149_ 159. [PubMed: 11672523]

11. Taverna SD, Li H, Ruthenburg AJ, Allis CD, Patel DJ. Nat Struct Mol Biol. 2007; 14:1025-1040. [PubMed: 17984965] 

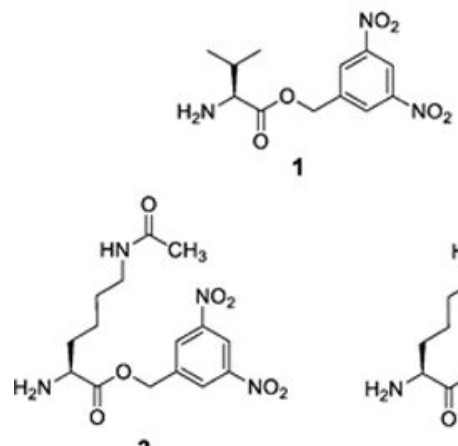
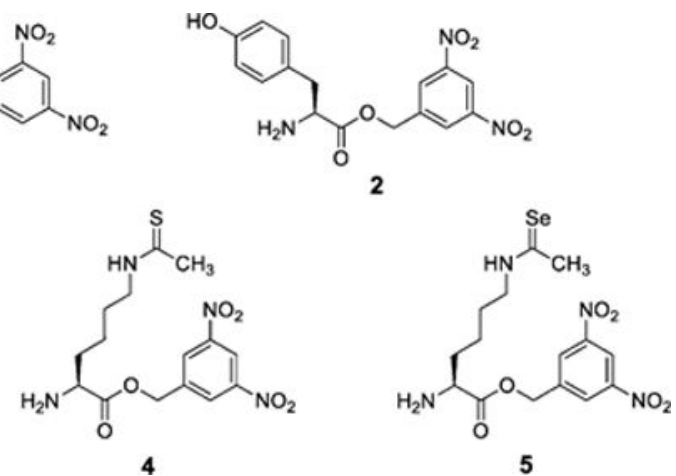

Abbildung 1.

Strukturen von Valin-3,5-dinitrobenzylester (Val-DBE, 1), Tyrosin-3,5-dinitrobenzylester (Tyr-DBE, 2), Acetyllysin-3,5-dinitrobenzylester (AcK-DBE, 3), Thioacetyllysin-3,5dinitrobenzylester (ThioAcK-DBE, 4) und Selenoacetyllysin-3,5-benzylester (SeAcK-DBE, 5). 
A

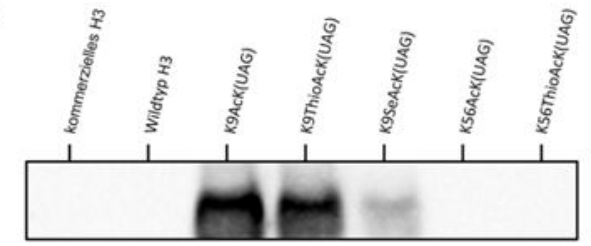

Anti-Acetylhiston $\mathrm{H} 3 \mathrm{~K} 9$

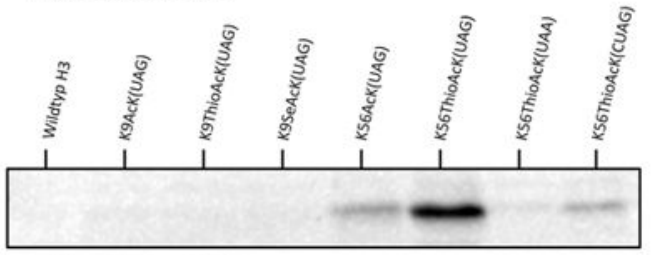

Anti-Acetylhiston H3K56

B

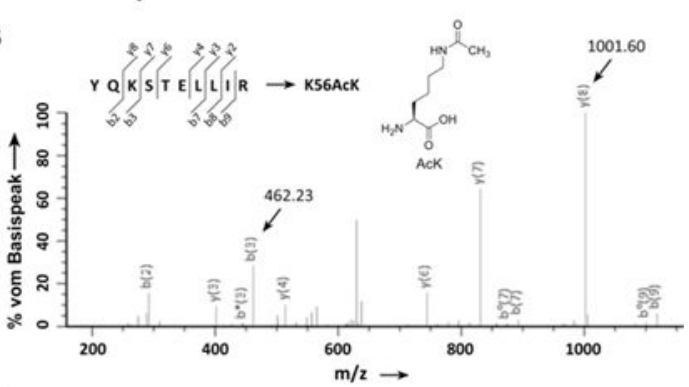

C
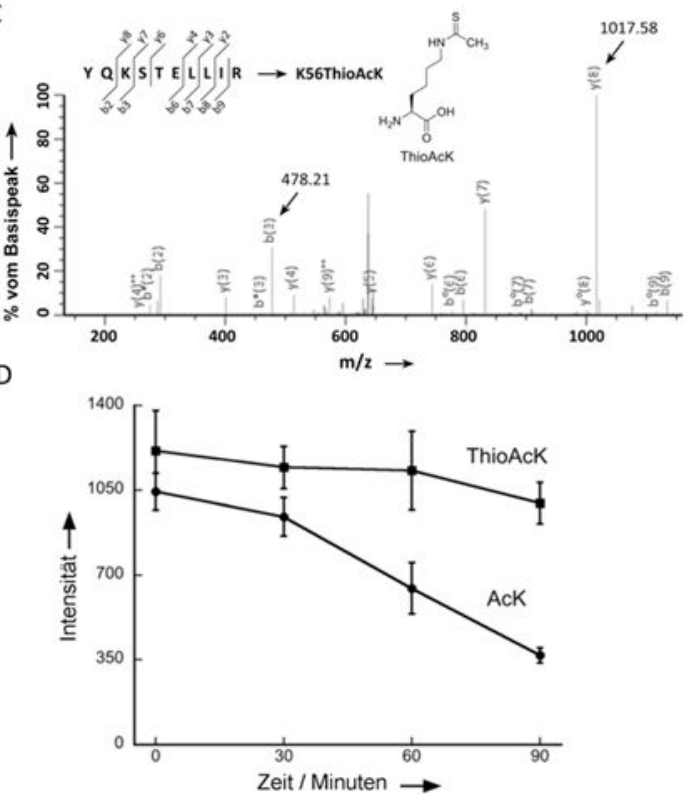

Abbildung 2.

Einbau von nkAS in humanes Histon H3. A) Western-Blot-Analyse der H3K9AcK- und H3K56AcK-Varianten mit Anti-Histon-H3K9AcK-und Anti-Histon-H3K56AcK-

Antikörpern. $2.5 \mu \mathrm{L}$ der PURE-Reaktionsmischung oder $1 \mu \mathrm{g}$ des kommerziell erworbenen H3-Proteins wurden in die Taschen des SDS-Polyacrylamidgels aufgetragen. B,C) MS/MSSpektren der tryptischen Peptide H3K56AcK (YQ-(AcK)STELLIR) (B) und H3K56ThioAcK (YQ(ThioAcK)STELLIR) (C). D) HDAC-Raten der Histon-Varianten H3K56AcK und H3K56ThioAcK über dem Verlauf über 90 Minuten. Deacetylierungs und 
Dethioacetylierungsreaktionen wurden durch Western-Blot-Analyse mit Anti-AcetyllysinHiston-H3K56-Antikörpern quantifiziert. Der Zeitpunkt $t=0$ min wurde für die Reaktionsmischung ohne Zugabe der Deacetylase festgelegt. Die Messwerte wurden über 3 Versuchsreihen gemittelt und die Fehlerbalken zeigen die \pm 1 Standardabweichungen an. 
A

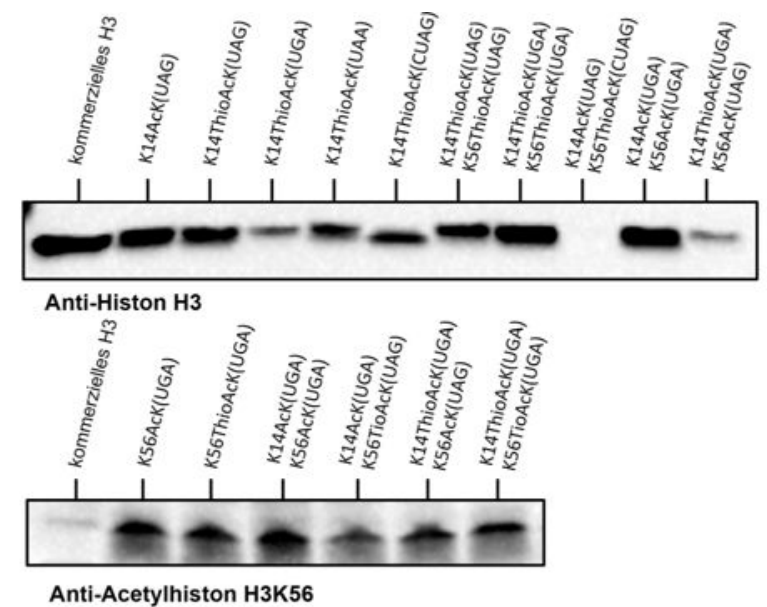

Anti-Acetylhiston H3K56

B
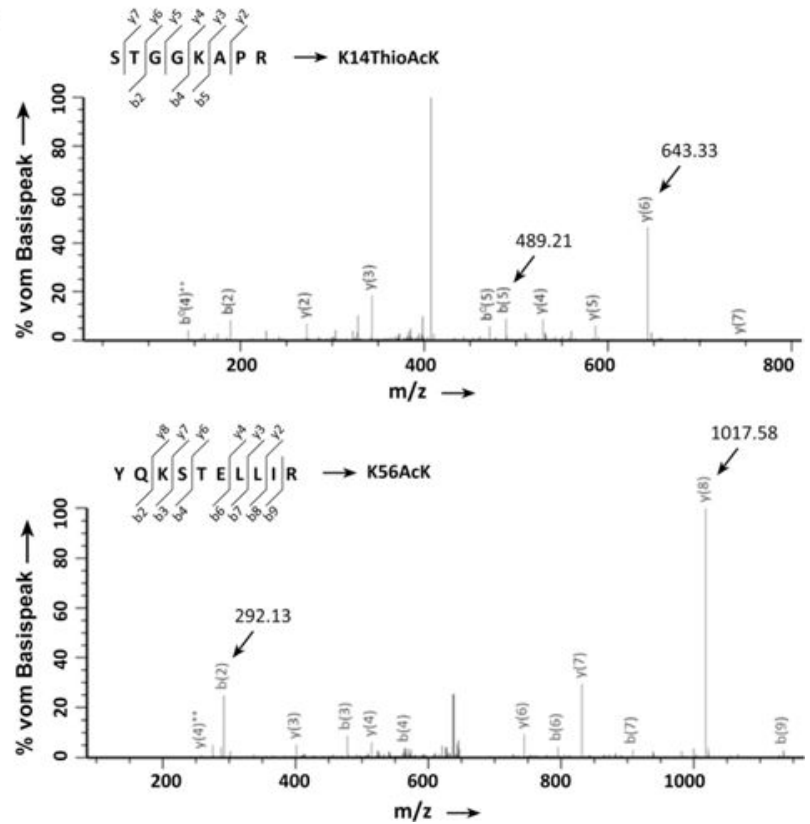

Abbildung 3.

Einbau von einem oder mehreren AcK- und ThioAcK-Bausteinen in die Histonvarianten H3K14 und H3K14/K56. A) Western-Blot-Analyse des Histon H3 mit Anti-Histon-H3Antikörpern bzw. Anti-56AcK-Histon-H3-Antikörpern. $5 \mu \mathrm{L}$ der PURE-Reaktionsmischung wurde jeweils in die Taschen des SDS-Polyacrylamidgels aufgetragen. Für die Spuren 1 dienten jeweils $1 \mu \mathrm{g}$ von kommerziell erworbenem Histon H3 zur Antikörper-Kontrolle. B) MS/MS-Spektren zweier tryptischer Peptide (STGG(ThioAcK)APR für die K14ThioAcKModifikation; YQ(AcK)STELLIR für die K56AcK-Modifikation), die aus der Hydrolyse der Histonvariante K14ThioAcK/K56AcK gewonnen wurden. 